\title{
Methylene blue promotes quiescence of rat neural progenitor cells
}

\section{Luokun Xie ${ }^{1}$, Gourav R. Choudhury' ${ }^{1}$ Jixian Wang ${ }^{1}$, Yong Park', Ran Liu' ${ }^{1}$, Fang Yuan ${ }^{2}$, Chun-Li Zhang ${ }^{3}$, Thomas Yorio ${ }^{1}$, Kunlin Jin ${ }^{1}$ and Shao-Hua Yang ${ }^{1,2 *}$}

1 Department of Pharmacology and Neuroscience, University of North Texas Health Science Center, Fort Worth, TX, USA

2 Department of Neurosurgery, Beijing Neurosurgical Institute, Beijing Tiantan Hospital, Capital Medical University, Beijing, China

${ }^{3}$ Department of Molecular Biology, University of Texas Southwestern Medical Center, Dallas, TX, USA

\section{Edited by:}

Rena Li, Roskamp Institute, USA

Reviewed by:

Eumorphia Remboutsika, Biomedical Sciences Research Center Alexander Fleming, Greece

Jiping Tang, Loma Linda University, USA

\section{${ }^{*}$ Correspondence:}

Shao-Hua Yang and Kunlin Jin, Department of Pharmacology and Neuroscience, University of North Texas Health Science Center, 3500 Camp Bowie Boulevard, Fort Worth, TX 76107, USA

e-mail: shaohua.yang@unthsc.edu; kunlin.jin@unthsc.edu
Neural stem cell-based treatment holds a new therapeutic opportunity for neurodegenerative disorders. Here, we investigated the effect of methylene blue on proliferation and differentiation of rat neural progenitor cells (NPCs) both in vitro and in vivo. We found that methylene blue inhibited proliferation and promoted quiescence of NPCs in vitro without affecting committed neuronal differentiation. Consistently, intracerebroventricular infusion of methylene blue significantly inhibited NPC proliferation at the subventricular zone (SVZ). Methylene blue inhibited mTOR signaling along with down-regulation of cyclins in NPCs in vitro and in vivo. In summary, our study indicates that methylene blue may delay NPC senescence through enhancing NPCs quiescence.

Keywords: methylene blue, neural progenitor cell, quiescence, proliferation, neurogenesis

\section{INTRODUCTION}

Methylene blue is a heterocyclic aromatic chemical compound that is used to treat malaria (Dormoi et al., 2013), methemoglobinemia (Zenk, 2001), and ifosfamide neurotoxicity (Patel, 2006). Recent studies have identified its potential neuroprotective action in ischemic stroke, Parkinson's disease (Wen etal, 2011), and Alzheimer's disease (Congdon et al., 2012). In addition, $\mathrm{MB}$ modulates metabolism as an alternative mitochondrial electron carrier and enhances mitochondrial oxidative phosphorylation (Wen etal., 2011; Poteet etal., 2012, 2013). MB can induce autophagy to protect neurons from serum deprivation (Xie et al., 2013). Metabolism is known to play a pivotal role in dictating whether a cell proliferates, differentiates, or remains quiescent (Shyh-Chang et al., 2013). Coincidentally, MB has been demonstrated to delay fibroblast senescence (Atamna et al., 2008) and inhibit glioblastoma cells proliferation (Poteet et al., 2013). However, whether MB has effects on NPCs has not been addressed.

Neural progenitor cells reside in distinct regions of adult mammalian brains and have the capacity for self-renewal and giving rise to three neural lineages through asymmetric cell division (Gage, 2000). NPCs are involved in the maintenance of normal brain homeostasis and participate in CNS (central nervous system) regeneration and repair (Barkho and Zhao, 2011; Liu and Rando, 2011). Stem cell exhaustion has been recognized as one of the hallmarks of aging (López-Otín et al., 2013). Depletion of

Abbreviations: CNS, central nervous system; DCX, doublecortin; GFAP, glial fibrillary acidic protein; MAP2, microtubule-associated protein 2; MB, methylene blue; NPCs, neural progenitor cells; SVZ, subventricular zone.
NPCs, possibly through loss of self-renewal capacity, has been indicated to be responsible for declining neurogenesis with age (Renault et al., 2009). Thus, maintenance of self-renewal and prevention of senescence is of great importance for longevity research and anti-aging therapeutics. In this study, we determined the effect of $\mathrm{MB}$ on the proliferation, self-renewal and differentiation of NPCs in vitro and in vivo. We demonstrated that MB inhibited proliferation and promoted quiescence of NPCs, but not affecting committed neuronal differentiation. In addition, we found that MB down-regulated mTOR and cyclins expression in NPCs.

\section{MATERIALS AND METHODS EXPERIMENTAL ANIMALS}

All experiments were conducted in compliance with institutional guidelines and NIH Guidelines for the Use of Animals. All animal procedures were approved by the University of North Texas Health Science Center Animal Care and Use Committee. Male SpragueDawley rats weighing $250-300 \mathrm{~g}$ were purchased from the Jackson Laboratory. Two mM MB (American Regent, Shirley, NY, USA) in $100 \mu \mathrm{l}$ of artificial cerebrospinal fluid was administered into the left lateral ventricle using Alzet osmotic minipumps $(0.5 \mu \mathrm{l} / \mathrm{h})$ for 7 days. Bromodeoxyuridine $(50 \mathrm{mg} / \mathrm{kg}$ BrdU, Sigma-Aldrich, St. Louis, MO, USA) was intraperitoneally injected twice a day during the first 3 days of $\mathrm{MB}$ treatment. All rats were euthanized and brains were collected for cryosectioning.

\section{NEUROSPHERE CULTURE AND IN VITRO MB TREATMENT}

Neurosphere cultures were performed following previous literature (Imura et al., 2003). Briefly, SVZ of mixed postnatal day 1 rats were digested with $0.25 \%$ Trypsin-EDTA (Invitrogen, Grand 
Island, NY, USA) at $37^{\circ} \mathrm{C}$ for $30 \mathrm{~min}$. Cells were dissociated by trituration and resuspended at 50,000 cells $/ \mathrm{ml}$ in Neurobasal medium (Invitrogen, Grand Island, NY, USA) supplemented with B27, $10 \mathrm{ng} / \mathrm{ml}$ fibroblast growth factor-2 (FGF-2) and $10 \mathrm{ng} / \mathrm{ml} \mathrm{epi-}$ dermal growth factor (EGF, both from Peprotech, Rocky Hill, NJ, USA). Culture medium in the presence or absence of $5 \mu \mathrm{M} \mathrm{MB}$ was changed every 3 days. To assess the neurosphere formation in serial passage assay, primary neurospheres were mechanically dissociated into single cell suspension. $1 \times 10^{4}$ NPCs in $1 \mathrm{ml}$ of culture medium were incubated for 7 days in 12-well suspension culture plates. The number of neurospheres was counted at day 7. Then serial subculture and neurosphere counting was repeated every 7 days, with $1 \times 10^{4}$ NPCs being seeded at the beginning of every passage. $\mathrm{MB}$ was added into cell suspension at the final concentration of $5 \mu \mathrm{M}$ at the time of plating cells. To assess NPC differentiation, neurosphere were triturated into single cells before plating onto poly-L-lysine-coated coverslips in Neurobasal medium supplemented with B27 free of FGF2 and EGF. MB $(5 \mu \mathrm{M})$ was added at $24 \mathrm{~h}$ after plating.

\section{APOPTOSIS ASSAY}

Neural progenitor cells were stained with propidium iodide (PI) and Annexin V-FITC (both from BD Biosciences, Franklin Lakes, NJ, USA) according to the manufacture's instruction. Cells were analyzed on a BD LSRII flow cytometer.

\section{CELL PROLIFERATION AND CELL CYCLE ANALYSIS}

For cell proliferation analysis, NPCs were stained with $5 \mu \mathrm{g} / \mathrm{ml}$ antibody against Ki67 (Abcam, Cambridge, MA, USA). For cell cycle analysis, NPCs were fixed with 70\% ethanol and stained with PI (40 $\mu \mathrm{g} / \mathrm{ml}$, BD Biosciences, Franklin Lakes, NJ, USA) according to the manufacture's instruction. DNA content was analyzed on a BD LSRII flow cytometer.

\section{IMMUNOCHEMISTRY}

Cultured cells were fixed with 4\% paraformaldehyde and stained with anti-MAP2 antibody (Santa Cruz, Dallas, TX, USA) followed by staining with Alexa Fluor 488-conjugated goat anti-rabbit IgG (Invitrogen, Grand Island, NY, USA). Fluorescent microscopy was conducted using an Axio Observer Z1 fluorescent microscope (Zeiss, Thornwood, NY, USA). On each slide, cells in five randomly selected microscopy fields were counted. For brain section staining, rats were anesthetized and perfused with neutral-buffered formalin. The brains were harvested and fixed in neutral-buffered formalin overnight before sinking in 30\% sucrose. Frozen sections $(20 \mu \mathrm{m})$ were prepared followed by staining with mouse anti-BrdU $\mathrm{mAb}$ and rabbit anti-Nestin antibody (both from Sigma-Aldrich, St. Louis, MO, USA). Sections were observed on a LSM 410 confocal microscope (Zeiss, Thornwood, NY, USA) to record the Z-stack images to confirm colocalization of Nestin and BrdUlabeled cells in the SVZ. Images were analyzed with the LSM Image Browser software. BrdU- and Nestin-positive and double-labeled cells in the SVZ, along the lateral walls of the lateral ventricles (beginning at $1.18 \mathrm{~mm}$ anterior to bregma), were counted in four sections per animal, spaced $120 \mu \mathrm{m}$ apart, Results were expressed as the average number of BrdU- and Nestin-positive cells in SVZ.

\section{WESTERN BLOT}

Cells or tissues were lysed in RIPA buffer. The following primary antibodies were used to detect the proteins of interest: (1) cyclin antibodies (Cyclin Antibody Sampler Kit, Cell Signaling, Danvers, MA, USA); (2) mTOR mAb, 4E-BP1 mAb, p70 S6 kinase mAb, and GAPDH mAb (Cell Signaling, Danvers, MA,USA); (3) mouse GFAP $m A b$ and rabbit DCX polyclonal antibody (Santa Cruz, Dallas, TX, USA). Protein bands were visualized and optical density was analyzed using a Biospectrum 500 imaging system (UVP, LLC, Upland, CA, USA).

\section{REAL-TIME RT-PCR}

Total RNAs were extracted using TRIzol and were reversely transcribed to cDNAs using SuperScript ${ }^{\circledR}$ III First-Strand Synthesis System according to the manufactures' instructions. Realtime PCR was performed using Fast SYBR ${ }^{\circledR}$ Green Master Mix on a 7300 Real-Time PCR System. Reagents and instruments were purchased from Invitrogen, Grand Island, NY, USA. Data was analyzed with 7300 system software. Primer sequences for each gene were shown as follows (Forward and reverse):

\section{Cond1: 5'-ACTGACAACTCTATCCGCCC-3'; 5'-TCTGTGCATGTTTGCGGATG- ${ }^{\prime}$. \\ Ccnd2: 5'-AAAGAGACCATCCCGCTGAC-3'; $5^{\prime}$-TGAAGTCGTGAGGGGTGACT- 3 '. \\ Ccnd3: 5'-GTACCGTCTGCCTGTTGCTG-3'; $5^{\prime}$-CAGGAAGTCGTGCGCAATCA- $3^{\prime}$. \\ Cone1: 5'-CCGACCTCTCAGTCCGATCC-3'; $5^{\prime}$-CCACGCACGCTGAATCATCA- $3^{\prime}$.}

Cone2: 5'-CAGGCCTATATATTGAGTTGGCG-3'; $5^{\prime}$-GGCTACTGCGTCTTGACATTC- $3^{\prime}$.

Cona1: 5'-CTGACCGTTCCAACCACCAA-3'; $5^{\prime}$-CTGCTGCTACCAAGGAAGGAA- $3^{\prime}$.

Ccna2: 5'-GCCAACTGCAAGGTAGAAAGC-3'; $5^{\prime}$-GCTCCAGCAATAAGCGATGG-3'.

Ccnb1: 5'-TAGATGCAGACGATGGTGGTG-3'; 5'-CAGTGACTTCACGACCCAGTA- $3^{\prime}$.

Ccnb2: 5'-TAAGGCGAGCCTCAAAAGCC-3'; $5^{\prime}$-ATGTCGTAGTCGACGAGGGT- 3 '.

Ccnb3: 5'-GGCACAATGCGAGAAGAACC-3'; $5^{\prime}$-ACTCTGTTAGGGAAGGGGGA-3'.

Map2: 5'-AAGCGGAAAACCACAGCAAC-3'; $5^{\prime}$-GGTCTTGGGAGGGAAGAACG-3'.

Nestin: 5'-GCACACCTCAAGATGTCCCT-3'; $5^{\prime}$-GAACCTCGTCCAGGTGTCTG-3'.

$D c x: 5^{\prime}$-TCACAGCATCTCCACCCAAC- $3^{\prime}$; $5^{\prime}$-GTCCATTCATCCGTGACCCT- $3^{\prime}$.

Gfap: 5'-GGAGAGGGACAATCTCACACAG-3'; 5'-TTCCTCTCCAGATCCACACGA-3'.

Gapdh: 5'-GATGGTGAAGGTCGGTGTGA-3'; $5^{\prime}$-TGAACTTGCCGTGGGTAGAG-3'.

\section{STATISTICAL ANALYSIS}

Data were analyzed and results were presented as mean $\pm \mathrm{SD}$. Student's $t$ test or one-way ANOVA was used for comparison of mean between the groups, and $p<0.05$ was considered statistically significant. 


\section{RESULTS}

\section{MB INHIBITS NPCs PROLIFERATION IN VITRO}

To determine the potential cytotoxicity of MB, NPCs were treated with $5 \mu \mathrm{M}$ MB for 6 days and apoptosis was analyzed. No significant change in apoptosis and necrosis was observed after treatment (Figure 1A). We then determined the effect of MB on NPC proliferation by Ki67 staining. The proportion of $\mathrm{Ki}^{+} 7^{+}$cells were significantly reduced at day 1 and day 3 after MB treatment, compared with control group (Figure 1B). On day 5 after treatment, NPC proliferation returned to the control level (Figure 1B). Consistently, cell cycle analysis with PI staining indicated that $\mathrm{MB}$ treatment induced significant G0/G1 arrest in NPCs at day 1 and day 3 post-treatment (Figure 1C).

\section{MB DOWN-REGULATES CYCLIN EXPRESSION IN NPCS IN VITRO}

Cyclins are a family of proteins that control cell progression through the cell cycle by activating cyclin-dependent kinases (Bloom and Cross, 2007; Lange and Calegari, 2010). Having observed the anti-proliferative effect of MB on NPCs, We then determined the effect of MB on cyclin expression in NPCs. Since Figure 1B showed that 1 day treatment had the most robust effect, we selected this time point to investigate. Consistent to its action on cell cycle, 24 h treatment with MB down-regulated various NPC cyclins at mRNA levels and/or protein levels. MB did not affect cyclin D1, D2, and D3 transcription, but significantly reduced the mRNA levels of cyclin E1, E2, A1, A2, B1, and B3, although there was an increase in cyclin B2 mRNA (Figure 2A). Western blot confirmed down-regulated expression of cyclin E1 and B1 after $\mathrm{MB}$ treatment (Figures 2B,C). In addition, protein levels of cyclin D1 and D2 were also down-regulated after MB treatment (Figures 2B,C), suggesting that $\mathrm{MB}$ inhibited translation or enhanced degradation of cyclin D1 and D2.

\section{MB ENHANCES NEUROSPHERE GENERATION IN SERIAL PASSAGE ASSAY}

Neural progenitor cells are able to maintain their relatively undifferentiated state through self-renewal under both steady state and pathological conditions. Self-renewal is essential for stem cells to maintain stem cell pool within organs and tissues (He et al., 2009). To determine whether the MB-induced NPC quiescence favors neurosphere formation, we firstly tested mRNA levels of neural markers to determine NPC differentiation in the suspension culture. We found that NPCs progressively up-regulated expression of DCX, MAP2, and GFAP even in suspension culture with growth factors. Nestin and Sox 2 expression were up-regulated from day 1 to day 3 but were reduced after day 3. MB maintained Nestin and Sox2 expression, while significantly inhibited expression of DCX, MAP2, and GFAP as compared with vehicle control (Figures 3A-E), suggesting MB restrained NPCs differentiation in suspension culture. We then performed serial passage assay to check the self-renewal capability of NPCs. We found that neurosphere number was decreased over serial passages in either group, while MB treatment generated more neurospheres from passage 3-6, suggesting that neurosphere generation was promoted by MB (Figure 3F). Consistent with less proliferation, MB decreased the total NPC number in each passage, with the most significant decrease

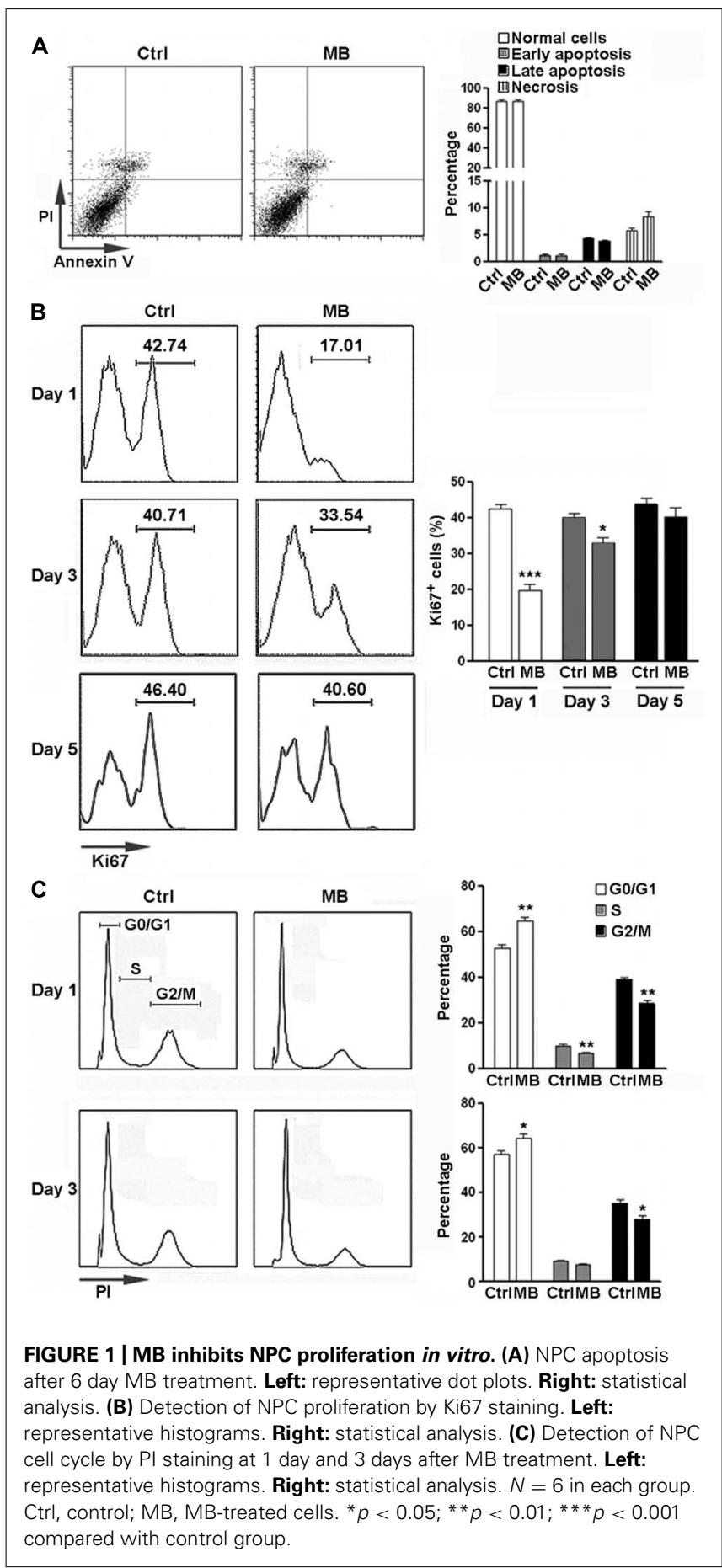

displayed in passage 3 and 4 (Figure 3G). Western blot assay confirmed lower expression of DCX and GFAP in MB-treated cells (Figure 3H), suggesting that differentiation was indeed inhibited by $\mathrm{MB}$.

\section{MB DOES NOT AFFECT COMMITTED NEURONAL DIFFERENTIATION FROM NPCs}

The other important function of NPCs is to differentiate into neurons and glia to sustain or repair the CNS structure. To 

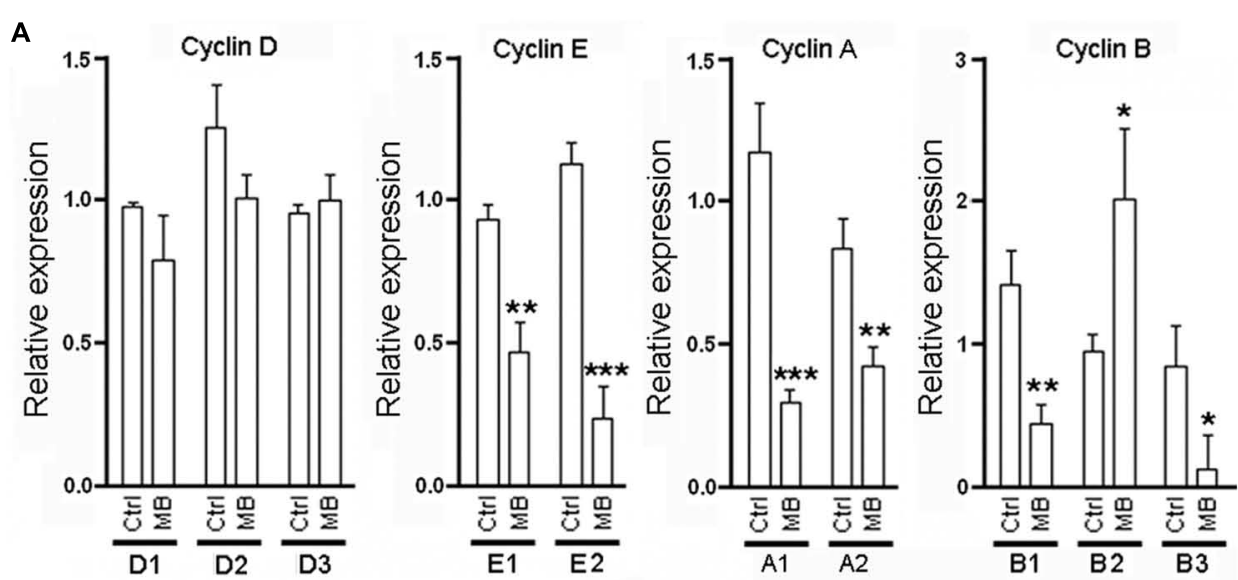

B

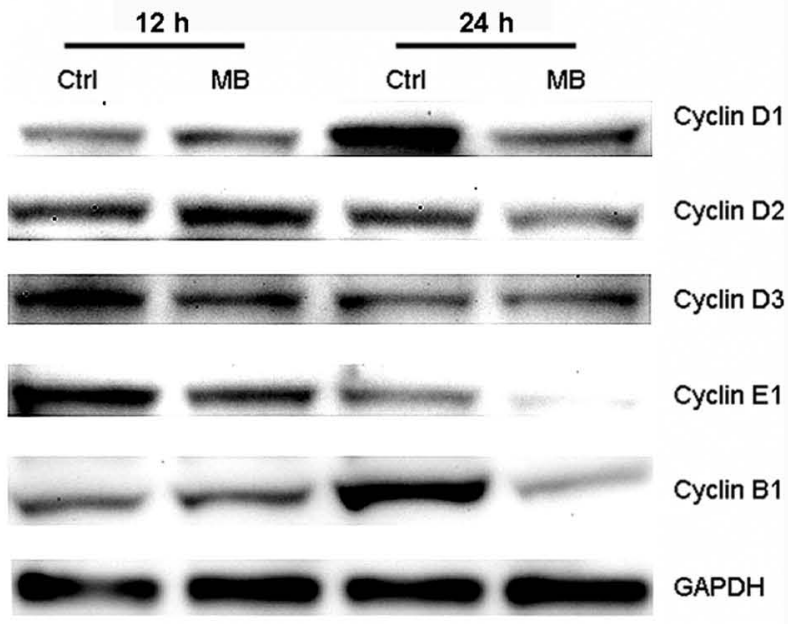

C Cyclin D1

Cyclin D2

Cyclin D3
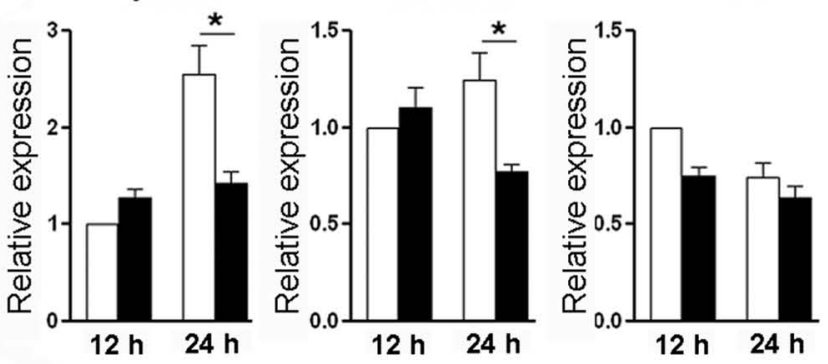

Cyclin E1

Cyclin B1
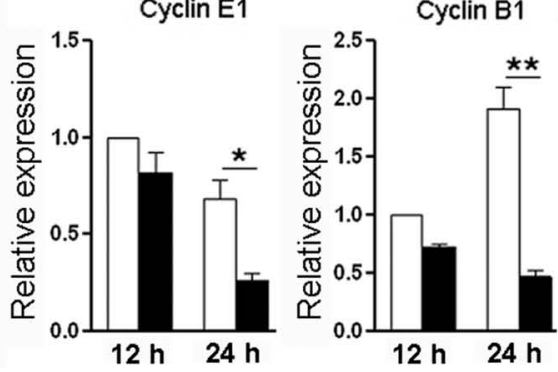

FIGURE 2 | MB down-regulates cyclin expression in NPCs.

(A) Detection of mRNA levels of cyclin $(D, E, A, B)$ in NPCs at $24 \mathrm{~h}$ after

MB treatment. (B) Western blot assay for cyclin expression in NPCs. This

is a representative of three independent experiments. (C) Statistical

analysis for cyclin protein levels. Expression level was normalized to each control group at $12 \mathrm{~h}$ after culture. $N=6$ in each group. Ctrl, control; MB, MB-treated cells. ${ }^{*} p<0.05 ;{ }^{* *} p<0.01 ;{ }^{* *} p<0.001$ compared with control group. 


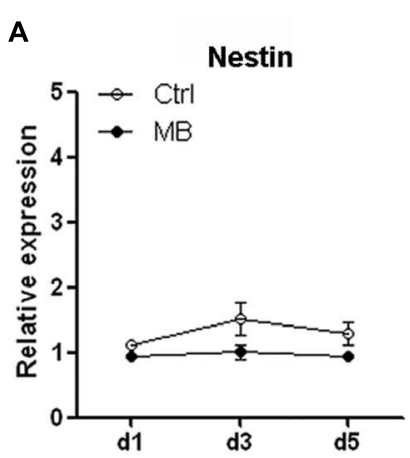

D

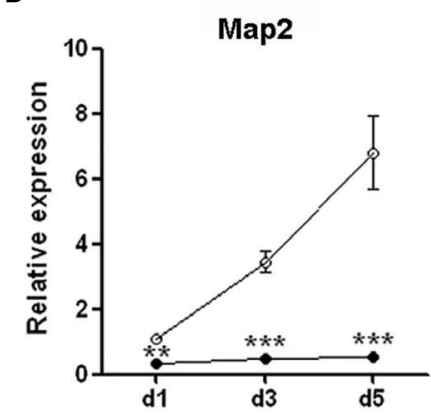

G

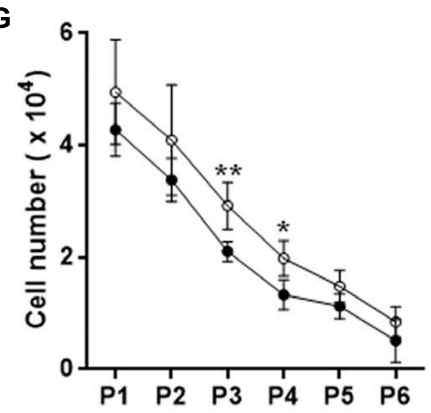

B

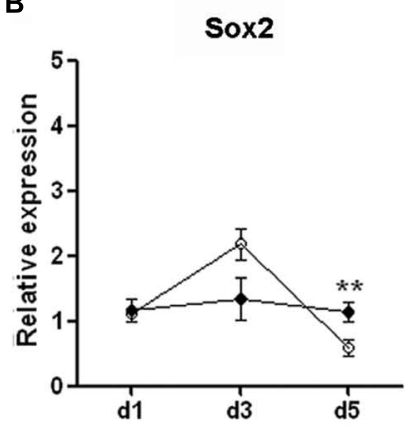

E

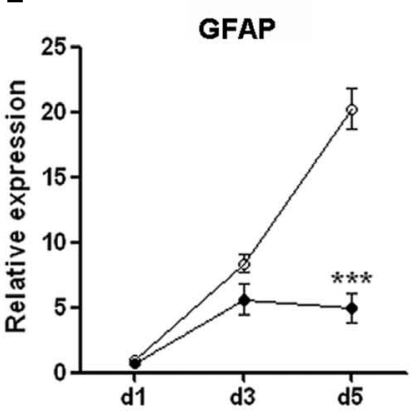

H

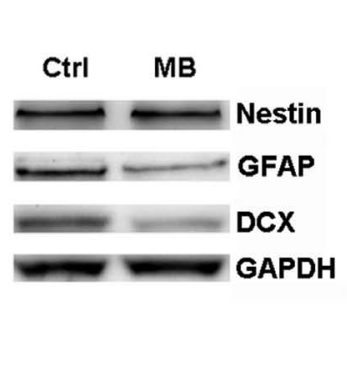

C

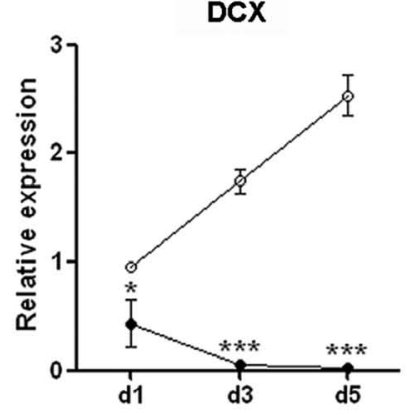

$\mathbf{F}$
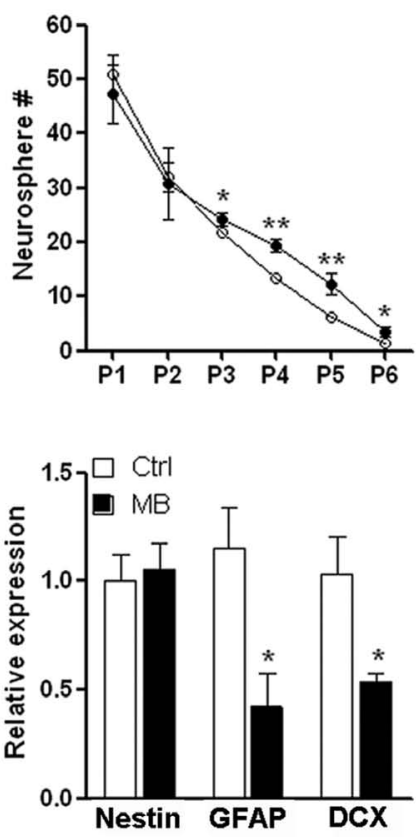

FIGURE 3 | MB maintains neurosphere formation capacity of NPCs. (A-E) Detection of mRNA levels of stem cell, neural and glial markers in passage 1 (P1) NPCs. N=3 in each group. (F) Serial passage analysis for neurosphere formation. $N=6$ in each group. (G) Total cell number counting at the end of each passage. Note that at the beginning of each passage, only $1 \times 10^{4}$ NPCs were seeded. (H) Western blot assay for Nestin, GFAP and DCX expression in P1 NPCs treated with MB for 6 days. Left, representative Western blot image. Right, statistical analysis for expression level of each protein. ${ }^{*} p<0.05 ;{ }^{* *} p<0.01 ;{ }^{* *} p<0.001$ compared with control group. test whether $\mathrm{MB}$ affected committed neuronal differentiation, we induced NPCs differentiation by withdrawing growth factors in adhesion culture for 7 days. Differentiation marker expressions were all robustly up-regulated while Nestin was significantly down-regulated on day 7 , suggesting that induction of differentiation was successful (Figure 4A). MB did not significantly alter the expression of MAP2, tubulin $\beta$-III and GFAP at 7 days after growth factors withdraw (Figure 4B). Consistently, immunofluorescence staining showed comparable MAP2 expression in control and MB-treated cells, indicating that MB did not affect committed neuronal differentiation (Figures 4C,D). Western blot assay also demonstrated comparable level of each differentiation marker between control and MB-treated groups (Figure 4E). Thus, it seems that $\mathrm{MB}$ does not affect committed neuronal differentiation from NPCs.

\section{MB INHIBITS TRANSCRIPTION OF mTOR, p70S6K, AND 4EBP1}

The close relationship between mTOR signaling and cell cycle progression has been elucidated elsewhere (Fingar et al., 2004). Based on the above data showing MB impedes cyclin D translation, we speculated that MB down-regulates mTOR signaling to modulate NPC proliferation. So we first determined mTOR activating phosphorylation in MB-treated NPCs. We did observe decreased mTOR phosphorylation at Ser 2448 after MB treatment. In addition, we found the protein level of total mTOR was also reduced (Figure 5A). Thus, the decreased phosphorylated mTOR is likely due to decreased total mTOR protein. Then we determined the expression of two target proteins of mTOR -p70S6K and 4EBP1- in the mTORC1 signal pathway. We found that $\mathrm{MB}$ robustly down-regulated protein levels of both p70S6K and 4EBP1 (Figures 5B,C). To test whether MB enhances 


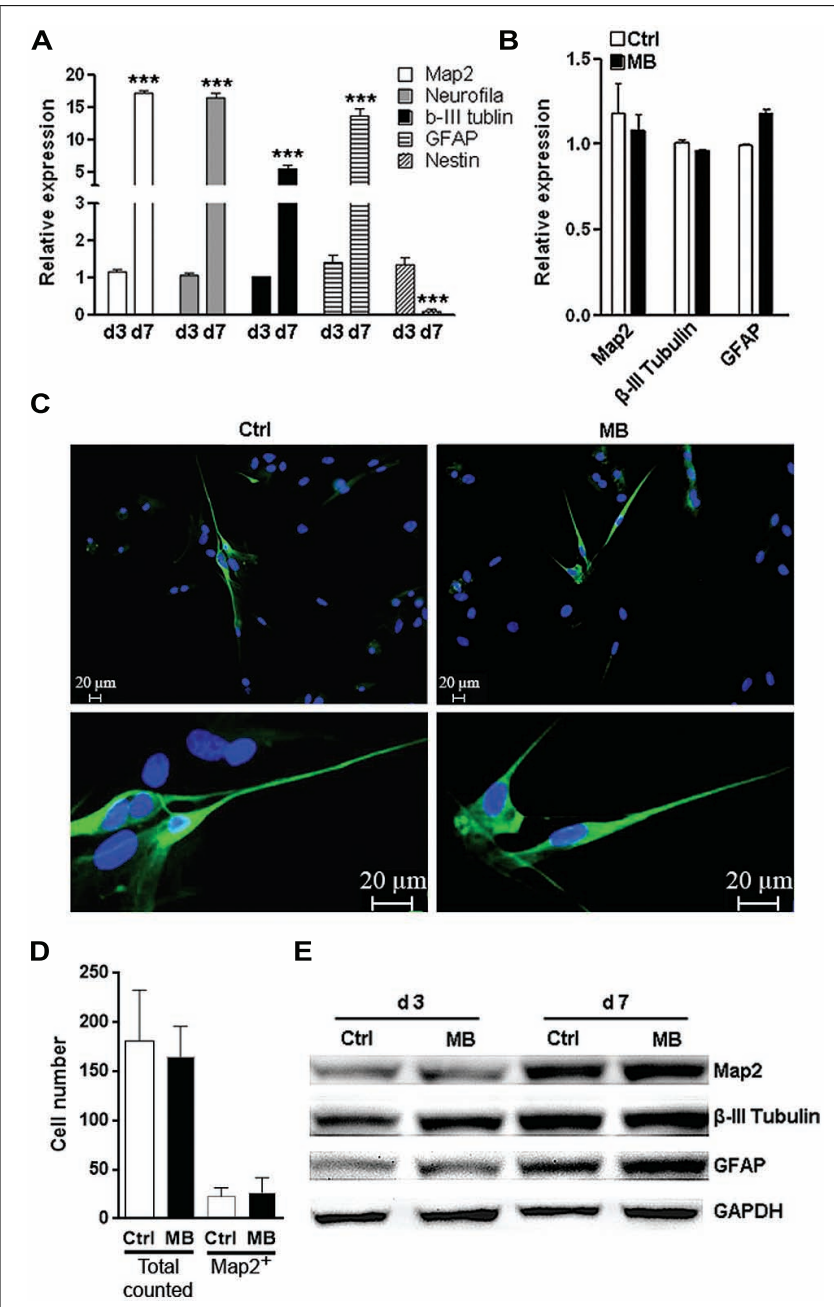

FIGURE 4 | MB does not impede committed neuronal differentiation. (A) Q-RTPCR analysis of the expression of differentiation markers in differentiated NPCs at 1 and 7 days after growth factors withdraw. $N=3$ per group. ${ }^{* *} p<0.001$ compared with day-1 differentiated NPCs. (B) Q-RTPCR for expression of differentiation markers in differentiated NPCs in the presence or absence of MB. $N=3$ per group. (C) Map2 staining for differentiated NPCs in the presence or absence of MB. Upper, $50 \times$; lower, $200 \times$. (D) Total cell number counting and Map2 ${ }^{+}$cell number in five microscopy fields $(50 \times)$. (E) Western blot assay for expression of differentiation markers in differentiated NPCs in the presence or absence of $\mathrm{MB}$. This is a representative of two independent experiments.

degradation of these proteins, NPCs were co-treated with proteasome inhibitor MG132. MG132 treatment did not increase protein levels of mTOR, p70S6K, and 4EBP1 in MB-treated NPCs, suggesting that protein degradation was not involved in $\mathrm{MB}$-induced down-regulation of these proteins (Figure 5D). Real-time PCR revealed that $\mathrm{MB}$ significantly decreased mRNA level of mTOR, p70S6K and 4EBP1 (Figure 5E). Thus, it seems MB inhibits mTOR transcription and in turn decreases mTOR protein, leading to reduction of cyclin D translation and inhibition of cell cycle entry.

\section{MB ALLEVIATES NPC PROLIFERATION IN VIVO}

To test whether MB also induces NPC quiescence in vivo, $\mathrm{MB}$ was intracerebroventricularly infused into adult rats for 7 days.

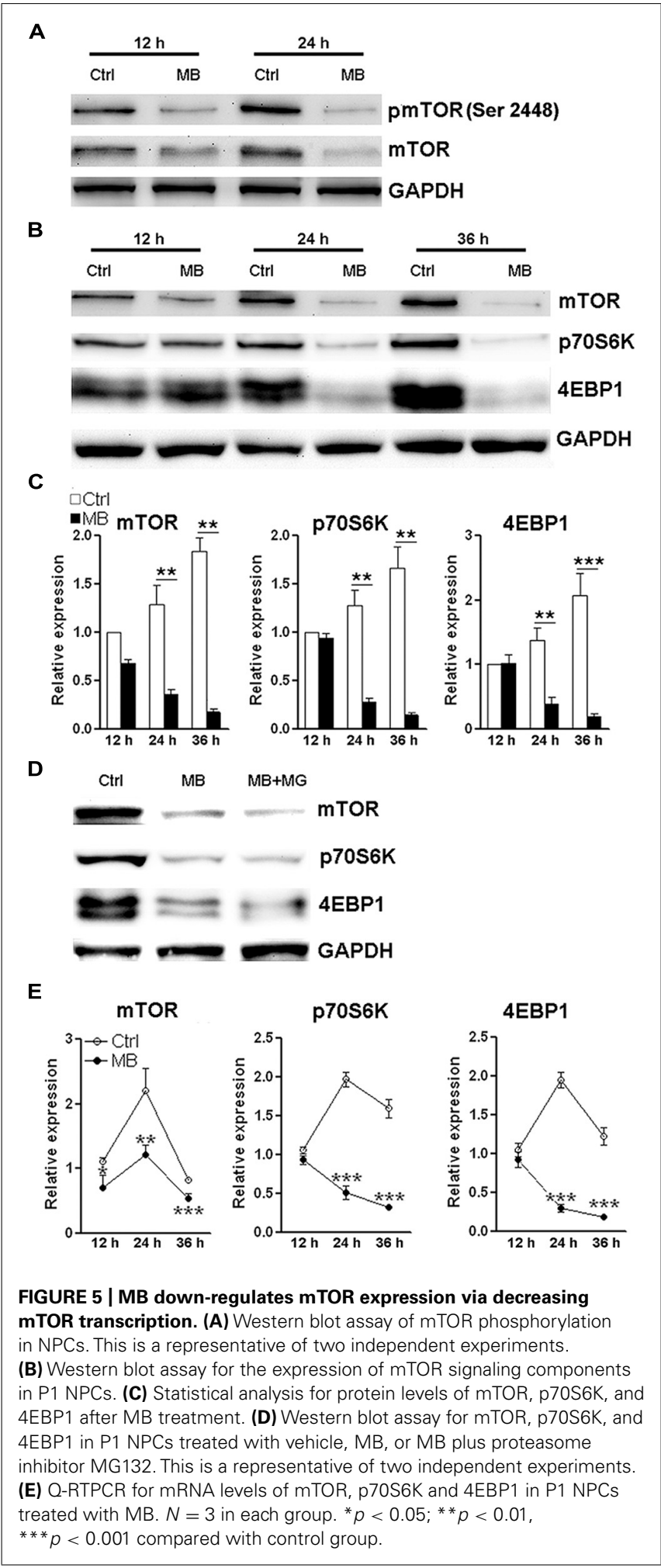

Immunofluorescence staining of Nestin and BrdU demonstrated less $\mathrm{Nestin}^{+} / \mathrm{BrdU}^{+}$cells at the SVZ in MB-treated rats, suggesting that MB inhibited NPC proliferation (Figures 6A,B). Consistent with our in vitro result, $\mathrm{MB}$ down-regulated $\mathrm{mTOR}$ and cyclin $\mathrm{D} 1$ expressions in the SVZ, while down-regulation of p70S6K was not 


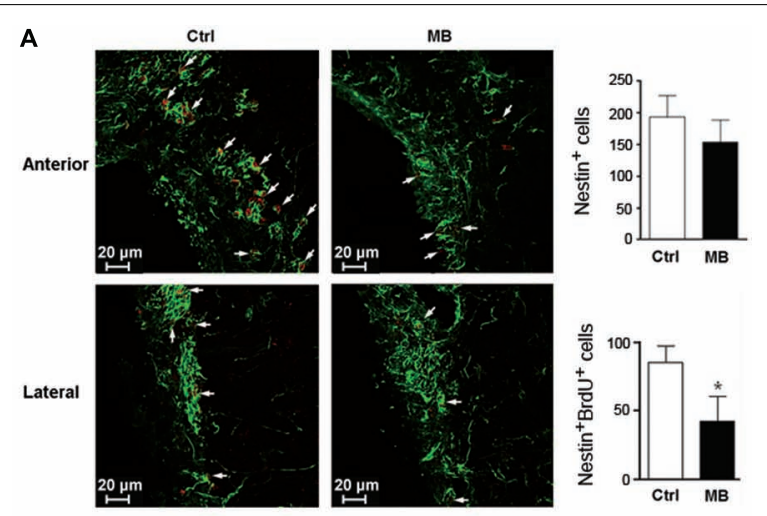

B
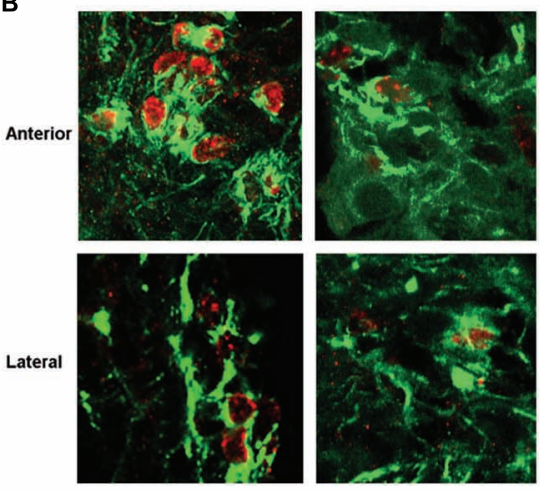

C
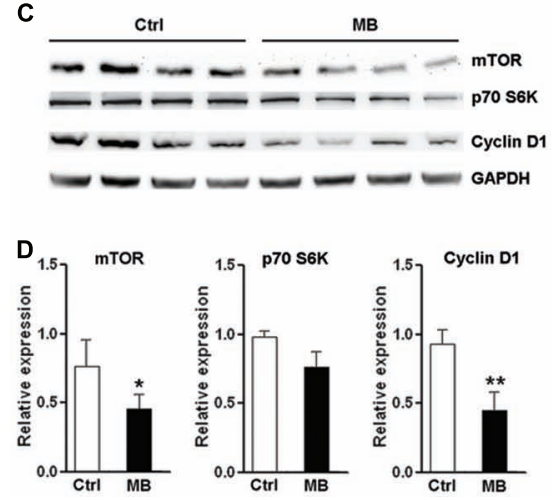

FIGURE 6 | MB restrains NPC proliferation in vivo. (A) Detection of NPC proliferation with Nestin and BrdU staining. Left: representative confocal microscopy images $(100 \times)$ of Nestin (green) and BrdU (red) at anterior and lateral part ventricular wall of SVZ in MB or vehicle treated rats. Arrows indicate Nestin ${ }^{+} / \mathrm{BrdU}^{+}$cells. Right: quantification of Nestin ${ }^{+}$cells and $\mathrm{Nestin}^{+} / \mathrm{BrdU}^{+}$cells in the SVZ. (B) Representative confocal microscopy images $(400 \times)$ of Nestin (green) and BrdU (red) at anterior and lateral part ventricular wall of SVZ. (C) Western blot assay for mTOR, p70S6K and cyclin D1 expression at SVZ in MB or vehicle-treated rats. (D) Statistical analysis for mTOR, p70S6K and cyclin D1 proteins at SVZ in MB or vehicle-treated rats. ${ }^{*} p<0.05 ;{ }^{*} p<0.01$ compared with control group.

as significant as that in in vitro assay (Figures 6C,D). Hence, we predicted that MB enhances the quiescence of NPCs by decreasing mTOR expression in vivo.

\section{DISCUSSION}

Neural progenitor cells are captivating given their capacity to differentiate into multiple brain cell types yet maintain their undifferentiated state through self-renewal by which a stem cell divides asymmetrically or symmetrically to generate one or two daughter stem cells that have the developmental potential of the mother cell. The ability to self-renewal is essential for stem cells to expand their numbers during development and to maintain stem cell pool within adult tissues (He et al., 2009). The slow replenishment of degenerating cells with newly generated neuronal cells under physiological conditions in vivo has suggested that neural stem cells basically rest in a state of quiescence, which allows them to maintain a balance between the ability to undergo selfrenewal and to differentiate without depleting the stem pool. The importance of stem cell quiescence for the long-term function of stem cells, including neural stem cells, has been demonstrated (Cheng et al., 2000; Kippin et al., 2005; Rera et al., 2011). Dysregulation and loss of quiescence often results in an imbalance in progenitor cell populations ultimately leading to stem cell depletion (Cheung and Rando, 2013). Thus, how to balance the NPCs proliferation/differentiation and quiescence to prevent premature senescence is of great interest in stem cell-related therapeutic strategies.

In this study we assessed the effect of MB on NPCs, focusing on two critical aspects of NPCs-quiescence and differentiation. Quiescence is an important property of adult stem cells for maintaining stem cell pool and preventing premature senescence. In vitro growth factor-driven serial passage assay continuously places neural stem cells under the proliferative stress so as to exhaust their neurosphere-forming capacity after several passages. MB slowed NPC proliferation evidenced by the reduction of Ki67 expression. MB treatment also induced NPC cell cycle arrest. As predicted, the inhibitory action of MB on NPCs was associated with an increase of neurosphere generation. Thus, our data indicated that MB induces NPCs into a relatively quiescent state. Noteworthily, $\mathrm{MB}$ treatment did not alter the expressions of mature neuronal markers when NPCs were cultured under differentiation condition. Thus, methylene treatment maintains NPC pool without affecting committed neuronal differentiation. The in vivo data proves that MB exerts similar inhibitory action on NPC proliferation at the SVZ. This is particularly beneficial for patients receiving $\mathrm{MB}$, since their neuronal development is unlikely to be affected.

Cyclins are a family of proteins that control cell progression through the cell cycle by activating cyclin-dependent kinases (Bloom and Cross, 2007; Lange and Calegari, 2010). MB down regulates various cyclins in NPCs. The pattern of MB-induced changes of cyclin expression provided further clues for the mechanism by which MB modulates NPC proliferation. Our data demonstrated that $\mathrm{MB}$ did not alter mRNA level of cyclin $\mathrm{D}$ while decreasing its protein level, suggesting that $\mathrm{MB}$ down-regulated translation of cyclin D. Expressions of most other cyclins such as cyclin E, B, and A were also decreased by MB. MB-induced cyclin D decrease might hamper G0/G1 transition and subsequent cell cycle progression, resulting in cell cycle arrest, proliferation inhibition and quiescence. The action of MB to reserve quiescent NPC pool may provide a pharmacological intervention to increase the longevity of neural stem cells.

The well-known regulatory factor of cyclin D translation is mTOR, which controls cell growth and metabolism. mTOR signaling controls cell growth by regulating both translation 
(Vadlakonda et al., 2013) and transcription (Wander et al., 2011) of cell cycle factors. The close relationship between mTOR signaling and cell cycle progression has been elucidated (Fingar et al., 2004). Previous studies has indicated the involvement of mTOR signaling in the maintenance of human embryonic stem cells (Zhou et al., 2009), NPCs (Han et al., 2013), hematopoietic stem cells (Chen et al., 2008), and cancer stem cells (Matsubara et al., 2013). mTOR signaling is also important for both self-renewal and differentiation of neural stem cells (Magri et al., 2011). mTORC1 inhibition with rapamycin has been shown to improve stem cell function in the epidermis, in the hematopoietic system, and in the intestine (Castilho et al., 2009; Chen et al., 2009; Yilmaz et al., 2012). Recent studies indicated that MB regulates cell signal transduction such as mTOR (Congdon et al., 2012) and AMPK signaling (Xie et al., 2013). In our study, MB inhibited the expression of mTORC1 signaling components, including mTOR, 4EBP1, and p70 S6 kinase. The association between mTORC1 inhibition and cyclin D decrease poses the possibility that MB induces NPC quiescence through blocking mTORC1 pathway. However, further studies are in need to elucidate how MB affects expression of mTORC1 components. Our data is the first showing the transcriptional regulation of mTOR by MB. To our surprise, we can hardly find previous publications demonstrating transcriptional regulation of mTOR. Most research focused on the activation of mTOR via serine phosphorylation and complex formation. A recent study indicates that Sox 2 suppress mTOR transcription, via binding at a region about $1.6 \mathrm{kbp}$ upstream of the transcription start site of the mTOR gene (Wang et al., 2013). Another study showed that mTOR is overexpressed with loss of the Rb1 family pathway (El-Naggar et al., 2009). Whether MB utilizes these two signal pathways to regulate mTOR transcription needs further investigation.

In summary, the current study has provided the first in vitro and in vivo evidence that MB treatment promotes NPC quiescence through induction of cell cycle arrest. The inhibitory action of MB on NPC proliferation is accompanied by an inhibition of mTOR signaling. We speculate that MB treatment could maintain NPCs in a relative quiescence stage without affecting the differentiation potential. Our finding warrants future studies to determine the long term action of MB on NPCs and functional outcome in healthy and neurodegenerative disease models.

\section{AUTHOR CONTRIBUTIONS}

Conducted experiments: Luokun Xie, Gourav R. Choudhury, Jixian Wang, Yong Park, and Ran Liu.

Data analysis: Luokun Xie.

Research design: Luokun Xie, Kunlin Jin, and Shao-Hua Yang.

Wrote or contributed to the writing of the manuscript: Luokun Xie, Chun-Li Zhang, Fang Yuan, Thomas Yorio, and Shao-Hua Yang.

\section{ACKNOWLEDGMENTS}

This work was supported by National Institutes of Health grantsNS054651 Shao-Hua Yang, NS088596 Shao-Hua Yang, R01AG021980 Kunlin Jin, AHA SDG16960084 Ran Liu, and National Natural Science Foundation of China Grant 81228009.

\section{REFERENCES}

Atamna, H., Nguyen, A., Schultz, C., Boyle, K., Newberry, J., Kato, H., et al. (2008). Methylene blue delays cellular senescence and enhances key mitochondrial biochemical pathways. FASEB J. 22, 703-712. doi: 10.1096/fj.079610com

Barkho, B. Z., and Zhao, X. (2011). Adult neural stem cells: response to stroke injury and potential for therapeutic applications. Curr. Stem Cell Res. Ther. 6, 327-338. doi: 10.2174/157488811797904362

Bloom, J., and Cross, F. R. (2007). Multiple levels of cyclin specificity in cell-cycle control. Nat. Rev. Mol. Cell Biol. 8, 149-160. doi: 10.1038/nrm2105

Castilho, R., Squarize, C., Chodosh, L., Williams, B., and Gutkind, J. (2009). mTOR mediates Wnt-induced epidermal stem cell exhaustion and aging. Cell Stem Cell 5, 279-289. doi: 10.1016/j.stem.2009.06.017

Chen, C., Liu, Y., Liu, R., Ikenoue, T., Guan, K. L., and Zheng, P. (2008). TSC-mTOR maintains quiescence and function of hematopoietic stem cells by repressing mitochondrial biogenesis and reactive oxygen species. J. Exp. Med. 205, 23972408. doi: $10.1084 /$ jem.20081297

Chen, C., Liu, Y., and Zheng, P. (2009). mTOR regulation and therapeutic rejuvenation of aging hematopoietic stem cells. Sci. Signal. 2, ra75. doi: 10.1126/scisignal.2000559

Cheng, T., Rodrigues, N., Shen, H., Yang, Y., Dombkowski, D., Sykes, M., et al. (2000). Hematopoietic stem cell quiescence maintained by $\mathrm{p} 21$ cip $1 /$ waf 1 . Science 287, 1804-1808. doi: 10.1126/science.287.5459.1804

Cheung, T. H., and Rando, T. A. (2013). Molecular regulation of stem cell quiescence. Nat. Rev. Mol. Cell Biol. 14, 329-340. doi: 10.1038/nrm3591

Congdon, E. E., Wu, J. W., Myeku, N., Figueroa, Y. H., Herman, M., et al. (2012). Methylthioninium chloride (methylene blue) induces autophagy and attenuates tauopathy in vitro and in vivo. Autophagy 8, 609-622. doi: 10.4161/auto. 19048

Dormoi, J., Briolant, S., Desgrouas, C., and Pradines, B. (2013). Efficacy of proveblue (methylene blue) in an experimental cerebral malaria murine model. Antimicrob. Agents. Chemother. 57, 3412-3414. doi: 10.1128/AAC.02381-12

El-Naggar, S., Liu, Y., and Dean, D. C. (2009). Mutation of the Rb1 pathway leads to overexpression of mTor, constitutive phosphorylation of Akt on serine 473, resistance to anoikis, and a block in c-Raf activation. Mol. Cell. Biol. 29, 57105717. doi: 10.1128/MCB.00197-09

Fingar, D. C., Richardson, C. J., Tee, A. R., Cheatham, L., Tsou, C., and Blenis, J. (2004). mTOR controls cell cycle progression through its cell growth effectors S6K1 and 4E-BP1/eukaryotic translation initiation factor 4E. Mol. Cell. Biol. 24, 200-216. doi: 10.1128/MCB.24.1.200-216.2004

Gage, F. (2000). Mammalian neural stem cells. Science 287, 1433-1438. doi: 10.1126/science.287.5457.1433

Han, J., Xiao, Z., Chen, L., Chen, B., Li, X., Han, S., et al. (2013). Maintenance of the self-renewal properties of neural progenitor cells cultured in three-dimensional collagen scaffolds by the REDD1-mTOR signal pathway. Biomaterials 34, 19211928. doi: 10.1016/j.biomaterials.2012.11.063

He, S., Nakada, D., and Morrison, S. J. (2009). Mechanisms of stem cell self-renewal. Annu. Rev. Cell Dev. Biol. 25, 377-406. doi: 10.1146/annurev.cellbio.042308.113248

Imura, T., Kornblum, H. I., and Sofroniew, M. V. (2003). The predominant neural stem cell isolated from postnatal and adult forebrain but not early embryonic forebrain expresses GFAP. J. Neurosci. 23, 2824-2832.

Kippin, T., Martens, D., and van der Kooy, D. (2005). p21 loss compromises the relative quiescence of forebrain stem cell proliferation leading to exhaustion of their proliferation capacity. Genes Dev. 19, 756-767. doi: 10.1101/gad. 1272305

Lange, C., and Calegari, F. (2010). Cdks and cyclins link G1 length and differentiation of embryonic, neural and hematopoietic stem cells. Cell Cycle 9, 1893-1900. doi: $10.4161 /$ cc. 9.10 .11598

Liu, L., and Rando, T. A. (2011). Manifestations and mechanisms of stem cell aging. J. Cell Biol. 193, 257-266. doi: 10.1083/jcb.201010131

López-Otín, C., Blasco, M., Partridge, L., Serrano, M., and Kroemer, G. (2013). The hallmarks of aging. Cell 153, 1194-1217. doi: 10.1016/j.cell.2013. 05.039

Magri, L., Cambiaghi, M., Cominelli, M., Alfaro-Cervello, C., Cursi, M., Pala, M., et al. (2011). Sustained activation of mTOR pathway in embryonic neural stem cells leads to development of tuberous sclerosis complex-associated lesions. Cell Stem Cell 9, 447-462. doi: 10.1016/j.stem.2011.09.008 
Matsubara, S., Ding, Q., Miyazaki, Y., Kuwahata, T., Tsukasa, K., and Takao, S. (2013). mTOR plays critical roles in pancreatic cancer stem cells through specific and stemness-related functions. Sci. Rep. 3, 3230. doi: 10.1038/srep 03230

Patel, P. N. (2006). Methylene blue for management of Ifosfamide-induced encephalopathy. Ann. Pharmacother. 40, 299-303. doi: 10.1345/aph. $1 \mathrm{G} 114$

Poteet, E., Choudhury, G. R., Winters, A., Li, W., Ryou, M. G., Liu, R., et al. (2013). Reversing the Warburg effect as a treatment for glioblastoma. J. Biol. Chem. 288 9153-9164. doi: 10.1074/jbc.M112.440354

Poteet, E., Winters, A., Yan, L. J., Shufelt, K., Green, K. N., Simpkins, J. W., et al. (2012). Neuroprotective actions of methylene blue and its derivatives. PLoS ONE 7:e48279. doi: 10.1371/journal.pone.0048279

Renault, V. M., Rafalski, V. A., Morgan, A. A., Salih, D. A., Brett, J. O., Webb, A. E., et al. (2009). FoxO3 regulates neural stem cell homeostasis. Cell Stem Cell 5 , 527-539. doi: 10.1016/j.stem.2009.09.014

Rera, M., Bahadorani, S., Cho, J., Koehler, C., Ulgherait, M., Hur, J., et al. (2011). Modulation of longevity and tissue homeostasis by the Drosophila PGC-1 homolog. Cell Metab. 14, 623-634. doi: 10.1016/j.cmet.2011. 09.013

Shyh-Chang, N., Daley, G. Q., and Cantley, L. C. (2013). Stem cell metabolism in tissue development and aging. Development 140, 2535-2547. doi: 10.1242/dev.091777

Vadlakonda, L., Pasupuleti, M., and Pallu, R. (2013). Role of PI3K-AKT-mTOR and Wnt signaling pathways in transition of G1-S phase of cell cycle in cancer cells. Front. Oncol. 3:85. doi: 10.3389/fonc.2013.00085

Wander, S. A., Hennessy, B. T., and Slingerland, J. M. (2011). Next-generation mTOR inhibitors in clinical oncology: how pathway complexity informs therapeutic strategy. J. Clin. Invest. 121, 1231-1241. doi: 10.1172/JCI44145

Wang, S., Xia, P., Ye, B., Huang, G., Liu, J., and Fan, Z. (2013). Transient activation of autophagy via Sox2-mediated suppression of mTOR is an important early step in reprogramming to pluripotency. Cell Stem Cell 13, 617-625. doi: 10.1016/j.stem.2013.10.005
Wen, Y., Li, W., Poteet, E. C., Xie, L., Tan, C., Yan, L. J., et al. (2011). Alternative mitochondrial electron transfer as a novel strategy for neuroprotection. J. Biol. Chem. 286, 16504-16515. doi: 10.1074/jbc.M110.208447

Xie, L., Li, W., Winters, A., Yuan, F., Jin, K., and Yang, S. (2013). Methylene blue induces macroautophagy through $5^{\prime}$ adenosine monophosphate-activated protein kinase pathway to protect neurons from serum deprivation. Front. Cell. Neurosci. 7:56. doi: 10.3389/fncel.2013.00056

Yilmaz, Ö. H., Katajisto, P., Lamming, D., Gültekin, Y., Bauer-Rowe, K., Sengupta, S., et al. (2012). mTORC1 in the Paneth cell niche couples intestinal stem-cell function to calorie intake. Nature 486, 490-495. doi: 10.1038/nature11163

Zenk, K. E. (2001). Use of methylene blue to treat methemoglobinemia in infancy. Neonatal Netw. 20, 62-68. doi: 10.1891/0730-0832.20.5.62

Zhou, J., Su, P., Wang, L., Chen, J., Zimmermann, M., Genbacev, O., et al. (2009). mTOR supports long-term self-renewal and suppresses mesoderm and endoderm activities of human embryonic stem cells. Proc. Natl. Acad. Sci. U.S.A. 106, 78407845. doi: 10.1073/pnas.0901854106

Conflict of Interest Statement: The authors declare that the research was conducted in the absence of any commercial or financial relationships that could be construed as a potential conflict of interest.

Received: 10 June 2014; accepted: 18 September 2014; published online: 07 October 2014.

Citation: Xie L, Choudhury GR, Wang J, Park Y, Liu R, Yuan F, Zhang C-L, Yorio T, Jin K and Yang S-H (2014) Methylene blue promotes quiescence of rat neural progenitor cells. Front. Cell. Neurosci. 8:315. doi: 10.3389/fncel.2014.00315

This article was submitted to the journal Frontiers in Cellular Neuroscience.

Copyright ( $) 2014$ Xie, Choudhury, Wang, Park, Liu, Yuan, Zhang, Yorio, Jin and Yang. This is an open-access article distributed under the terms of the Creative Commons Attribution License (CC BY). The use, distribution or reproduction in other forums is permitted, provided the original author(s) or licensor are credited and that the original publication in this journal is cited, in accordance with accepted academic practice. No use, distribution or reproduction is permitted which does not comply with these terms. 